\title{
Voyager probing Dark Matter
}

\author{
Mathieu Boudaud* \\ LPTHE, CNRS \& Sorbonne Université, 4 Place Jussieu, Paris - France
}

E-mail: boudaudelpthe.jussieu.fr

The famous spacecraft Voyager-1 has crossed the heliopause in 2012, and is now exploring the local interstellar medium. This opens up a new avenue to probe the dark matter via cosmic ray electrons and positrons in the sub-GeV energy range. We combined the constraints from the Voyager-1 and AMS-02 data to get novel and robust limits covering a very extended dark matter particle mass range, from $\mathrm{MeV}$ to $\mathrm{TeV}$. For velocity dependent annihilation processes ( $p$-wave), we make use of the Eddington method to compute the phase space distribution function of dark matter particles from the most recent constrained mass model of the Galaxy. Primordial black holes are alternative and also well-motivated candidates for the dark matter. Black holes with a mass smaller than $\sim 10^{17} \mathrm{~g}$ are expected to inject electrons and positrons in the Galaxy via Hawking radiation. We show that Voyager-1 is sensitive to signatures from such black holes and we derive novel constraints on the contribution of primordial black holes to the dark matter in this mass window.

36th International Cosmic Ray Conference -ICRC2019-

July 24th - August 1st, 2019

Madison, WI, U.S.A.

${ }^{*}$ Speaker. 


\section{Introduction}

One of the most pressing questions in current cosmology is the nature of the dark matter (DM) that constitutes about $26 \%$ of the total energy-matter content of the Universe. Thermal DM particles is one of the most appealing DM scenario owing to its simplicity and to the fact that it can be experimentally or observationally tested. A prototypical candidate is the WIMP (weakly-interacting massive particle), which is currently actively searched for by a series of experiments. Primordial black holes (PBHs) are also well-motivated DM candidates, and this scenario has recently come back to the attention of the community. DM particles and PBHs are expected to inject CRs in the Galaxy. The former through annihilation or decay and the later via the Hawking radiation. Interstellar sub-GeV CRs are shielded by the solar magnetic field (the so-called solar modulation effect) such that they cannot reach detectors orbiting the Earth. Voyager-1 has crossed the heliopause during the summer 2012, and since then, has taken the first ever data of sub-GeV interstellar CRs. We make use of the $e^{ \pm}$Voyager-1 data from end 2012 [1] to probe the local abundance of DM whether it is in the form of annihilating (or decaying) particles or PBHs. On the one hand, we derive limits on the DM particles annihilation cross-section ( $s$-wave and $p$-wave) or decay lifetime $[2,3]$. On the other hand, we derive limits on the fraction of DM in the form of PBHs [4].

\section{Dark matter distribution and CRs transport}

We consider two spherically symmetric DM halos: a Navarro-Frenk-White (NFW) halo scaling like $1 / r$ in the center of the Galaxy and a cored halo featuring a constant density at small galactocentric radii. We use the kinematically constrained parameters provided in Table 6 of Ref. [6], where the DM density at the Sun position $R_{\odot} \simeq 8.2 \mathrm{kpc}$ is $\rho_{\odot} \simeq 0.4 \mathrm{GeV} / \mathrm{cm}^{3}$, and, for the cored profile, the core radius is $7.7 \mathrm{kpc}$.

The transport of CRs in the Galaxy is described by the phenomenological diffusion model described in Ref. [7] and extended for sub-10 GeV $e^{ \pm}$while accounting for all propagation processes by the pinching method [8]. The transport of sub-GeV $e^{ \pm}$is dominated by energy losses through ionisation of the interstellar medium. Therefore, $e^{ \pm}$measured by Voyager-1 are produced in the vicinity $(\sim 1 \mathrm{kpc})$ of the Solar System. We make use of two benchmark sets of transport parameters, dubbed hereafter models $A$ and $B$. Model $A$ is the model MAX of Refs. [7, 9]. For model $B$, we adopt the best fit parameters of Ref. [10], which makes use of the new AMS-02 B/C data to update the propagation parameters. Reference [10] obtained indications on the size of the magnetic halo $L>4.1 \mathrm{kpc}$ from the AMS-02 $e^{+}$flux. Hence, we will vary $L=4.1 \rightarrow 20 \mathrm{kpc}$ for model $B$. Model $A$ features a strong diffusive reacceleration, while there is none in model $B$. Since sub-GeV CRs $e^{ \pm}$are more sensitive to reacceleration than CR nuclei, we anticipate that the flux of $e^{ \pm}$will be drastically different for $A$ and $B$. The two models are therefore quite diverse and allow to quantify the impact of the CR propagation uncertainty on our results.

\section{Dark matter particles}

$\mathrm{DM}$ particles at the GeV-TeV scale are actively hunted thanks to direct and indirect detection techniques. However, the sub-GeV mass ranges are much less constrained and represent very 
interesting windows yet to be explored. The annihilation rate of DM particles is proportional to the average velocity-weighted annihilation cross section $\langle\sigma v\rangle$. In most cases, the annihilation cross section can be expanded in powers of $x^{-1} \equiv\left(m_{\chi} / T\right)^{-1} \propto v^{2} \ll 1$ where $m_{\chi}$ and $T$ are the DM mass and temperature, respectively. Making the units explicit, we may write this expansion as

$$
\begin{aligned}
\langle\sigma v\rangle & =\langle\sigma v\rangle_{s \text {-wave }}+\langle\sigma v\rangle_{p \text {-wave }}+\text { higher orders } \\
& =\sigma_{0} c+\sigma_{1} c\left\langle\frac{v_{\mathrm{r}}^{2}}{c^{2}}\right\rangle+\mathscr{O}\left(\frac{v_{\mathrm{r}}^{4}}{c^{4}}\right)
\end{aligned}
$$

where $\sigma_{0}$ and $\sigma_{1}$ are model-dependent cross-section terms that encode the DM particle interaction properties, $v_{\mathrm{r}} \ll c$ is the relative DM speed (in a 2-particle system), $c$ is the speed of light, and \langle\rangle denotes an average over the velocity distribution. This form is particularly well suited to consistently compare the constraints coming from very different probes. The speed-independent term $\propto \sigma_{0}$ is called $s$-wave annihilation in analogy with the partial-wave expansion technique. The next annihilation term $\propto \sigma_{1}$ is called $p$-wave annihilation.

In the $p$-wave annihilation case, the cross section has an explicit relative-speed dependence which is itself expected to vary across the Galactic halo. The $p$-wave signal depends on the velocity distribution function (DF) of DM particles in the system of interest. We adopt the Eddington inversion method, which relates the phase-space DF of DM particles to their mass density profile and the total potential of the Milky Way (including baryons) - see Ref. [11]. This approach allows us to describe isotropic as well as anisotropic systems, assuming spherical symmetry for the dark halo and the total gravitational potential. Ref. [11] noticed that a fully cored profile with the inner slope $\gamma=0$ would break necessary conditions for dynamical stability of the DF. We therefore use for the core profile an inner slope $\gamma=0.25$. Disregarding stability of the DF and forcing $\gamma=0$ would anyway provide results very similar to $\gamma=0.25$, which is therefore a very conservative case. To account for uncertainties in the DM anisotropy, we considered both isotropic and anisotropic DFs. In the latter case, we explore a wide range of possibilities by using both the radially anisotropic Osipkov-Merritt (OM) model with an anisotropy radius $r_{a}$ set to the scale radius $r_{s}$ of the DM halo profile, and a tangentially anisotropic model with a constant anisotropy parameter $\beta=-0.3$.

Template predictions for the DM-induced $e^{ \pm}$fluxes are shown in the left panel of Fig. 1, considering DM masses of $10 \mathrm{MeV}(10 \mathrm{GeV})$ annihilating into $e^{+} e^{-}(b \bar{b}$, respectively) for $s$-wave annihilation. In both cases, $e^{+} \mathrm{s}$ and $e^{-} \mathrm{s}$ share the same injection spectrum and experience the same propagation history, such that $e^{ \pm}$predictions can be compared to the AMS- $02 e^{+}$data by multiplying the latter by two. We reported our results for propagation models $A$ and $B$, and for the NFW and cored halos. Regarding propagation A, reacceleration effects dominate at low energy. This is clear for the $e^{+} e^{-}$channel when comparing predictions between model $A$ (strong reacceleration) and model $B$ (weak reacceleration) in the latter case, the $e^{ \pm}$flux is suppressed beyond the maximal injected energy set by $m_{\chi}$, while in the former case, low-energy $e^{ \pm} \mathrm{s}$ are reaccelerated beyond $m_{\chi}$ : $\mathrm{DM}$ annihilation-induced $e^{ \pm} \mathrm{S}$ could be observed at energies higher than $m_{\chi}$, which makes the $\mathrm{GeV}$ data also relevant to constrain sub-GeV DM candidates.

In the right panel of Fig. 1, we present our limits for several annihilation channels conservatively assuming propagation model $B, \phi=830 \mathrm{MV}$ for the solar modulation of the AMS-02 data, and the NFW halo. 
Limits on the $p$-wave cross section are shown in Fig. 2 assuming a full annihilation in $e^{+} e^{-}$. The left (right) panel corresponds to the cored (NFW) halo profile. We also show complementary bounds obtained with the CMB [12,13] (purple) rescaled to the latest Planck results [14], the highredshift intergalactic medium (IGM) temperature [13] (orange), the diffuse EGB [15, 16] (red), and gamma-ray observations of MW-satellite dwarf galaxies [17] (dark green curve). The CMB bound extrapolates the one obtained for $s$-wave annihilation by assuming a Maxwell-Boltzmann DF with a temperature at redshift $\sim 600$ that depends on the WIMP kinetic decoupling temperature $T_{\mathrm{kd}}$. We adopt two extreme values for the ratio $x_{\mathrm{kd}}=m_{\chi} / T_{\mathrm{kd}}, 10^{2}$ and $10^{4}$, to cover most of the relevant parameter (the CMB limit is $\propto x_{\mathrm{kd}}^{-1}$ ).
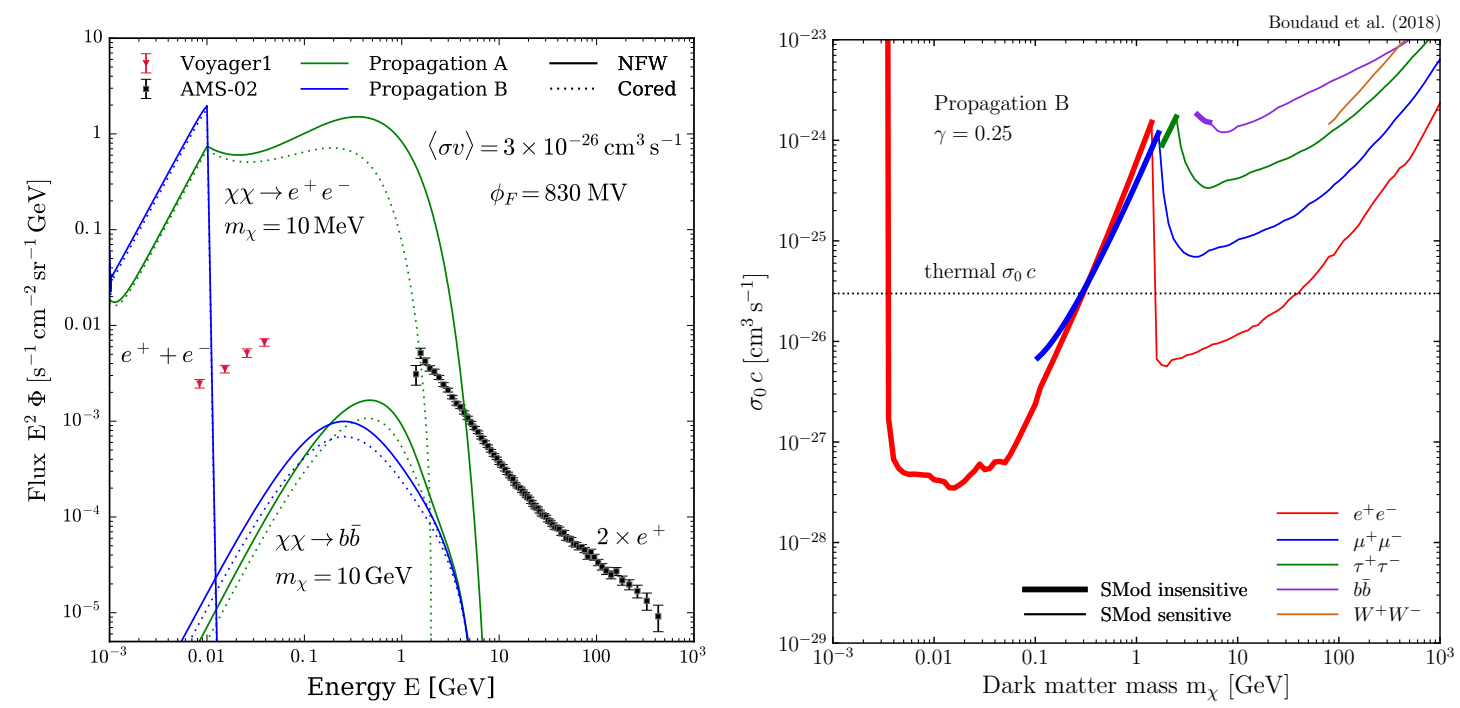

Figure 1: Left: predictions $\left(e^{ \pm}\right)$for 2 template cases: a $10 \mathrm{MeV}$ WIMP annihilating into $e^{+} e^{-}$, and a 10 GeV WIMP annihilating into $b \bar{b}$. AMS-02 $e^{+}$data are multiplied by a factor of 2 to compare with the $e^{ \pm}$ primaries. Propagation models $A$ and $B$, and the NFW and cored DM halo models were used. Right: limits for different annihilation final states, assuming configuration $B$-cored-830 MV.

\section{Primordial black holes}

PBHs would be generated in the early Universe when sufficiently large density perturbations in the primordial plasma collapse gravitationally. If they are formed early enough, the material of which they are made is subtracted very early on from the baryonic budget, and therefore they are not subject to the cosmological constraints from primordial nucleosynthesis and the CMB. In general terms, the expected mass of a PBH is connected to the time $t$ at which it was created, $M \sim c^{3} t / G \simeq 10^{15}\left(t / 10^{-23} \mathrm{~s}\right) \mathrm{g} \simeq 5 \times 10^{-19}\left(t / 10^{-23} \mathrm{~s}\right) M_{\odot}$, where $c$ is the speed of light, $G$ is the Newton constant, and $M_{\odot} \simeq 2 \times 10^{33} \mathrm{~g}$ is the mass of the Sun. Realistic production mechanisms predict not just a unique mass for all PBHs but rather an extended mass function. Whether some windows still exist in which PBHs (of fixed mass or distributed on a range of masses) can constitute $100 \%$ of the DM is currently subject to an intense debate. PBHs with $M \lesssim 4 \times 10^{14} \mathrm{~g}$ are ruled out because they are subject to Hawking evaporation, which would have made them disappear by now. 

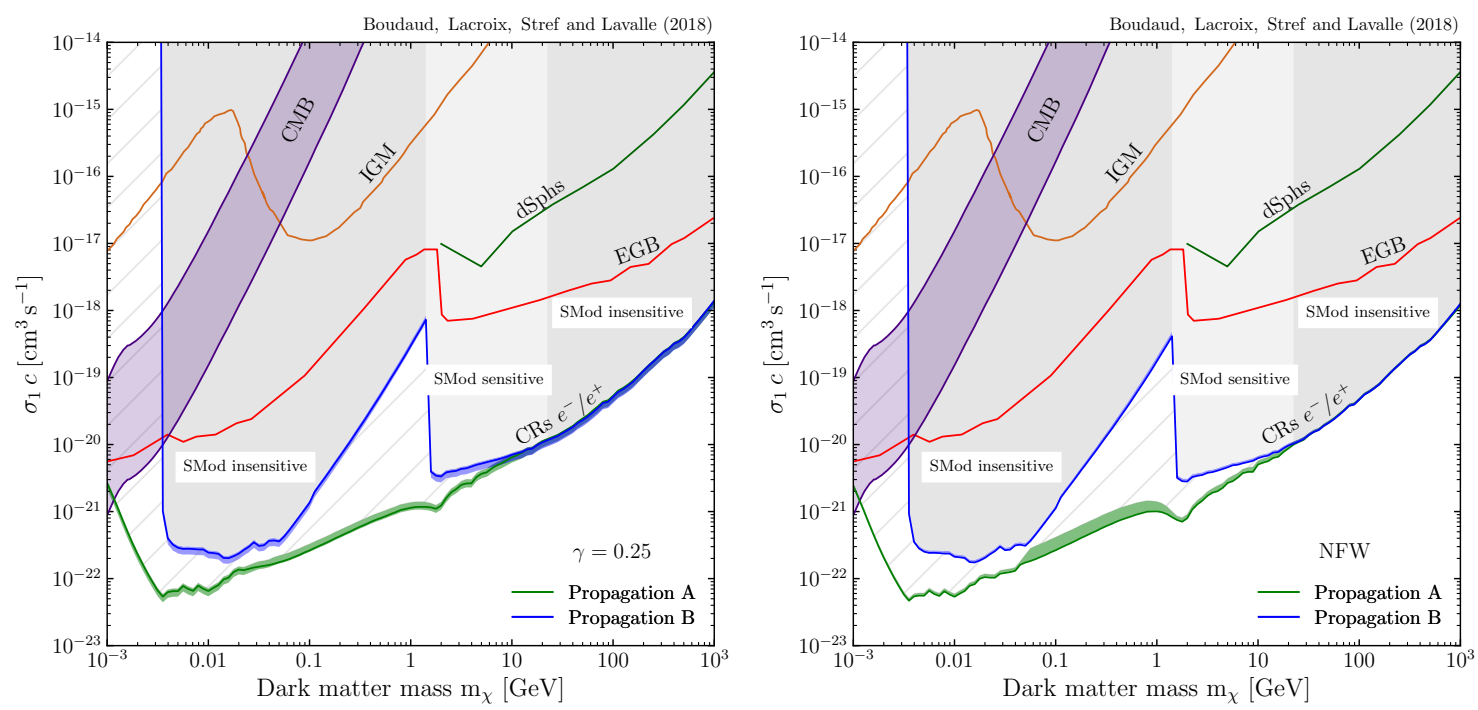

Figure 2: Limits on the $p$-wave cross section as a function of the DM mass $m_{\chi}$, in the $100 \% \chi \bar{\chi} \rightarrow e^{+} e^{-}$ channel, for a high-reacceleration propagation model (model $A$ - green curve) or a reacceleration-less, very conservative, propagation model (model $B$ - blue curve). Uncertainty bands account for uncertainties in the anisotropy of the DM velocity DF. Also shown are the limits obtained with the CMB [12, 13, 14], IGM [13], EGB [15, 16], and gamma-ray observations of dwarf galaxies [17]. Left: cored-like DM density profile with $\gamma=0.25$ [18]. Right: cuspy DM density profile with $\gamma=1$ [18].

For $4 \times 10^{14} \lesssim M \lesssim 10^{17} \mathrm{~g}$, PBHs are Hawking evaporating right now, emitting particles with a characteristic spectrum centered around tens of MeV. Indeed, constraints have been derived in the past using extragalactic gamma-ray background (EGB) observations [19]. While powerful, such constraints do not test the local DM density but rather its average extragalactic distribution. Instead, Voyager-1 is sensitive to $e^{ \pm}$radiated by PBHs in the Solar System vicinity. This will allow us to impose novel constraints on the fraction of the DM in the form of PBHs based on local measurements.

Assuming all DM of the Galaxy is made of single-mass PBHs (monochromatic mass function), we represent in the left panel of Fig. 3 the flux of $\left(e^{+}+e^{-}\right)$at the solar position produced by radiating PBHs with masses $10^{15}, 10^{16}$ and $10^{17} \mathrm{~g}$. The blue (red) curves are obtained from propagation model A (model B) whereas the solid (dotted) lines are obtained using the NFW (cored) DM halo profile. We thus use the Voyager- $1 e^{ \pm}$data to constrain the contribution of $\mathrm{PBHs}$ to the DM density in the Galaxy. The maximum fraction $f=\rho_{\mathrm{PBH}} / \rho_{\mathrm{DM}}$ is determined by requiring that the flux of $e^{ \pm}$emitted by PBHs does not overshoot any data point by more than $2 \sigma$. The limits for a monochromatic mass distribution are represented by the solid lines in the right panel of Fig. 3 . The blue (red) solid line is obtained with model $A$ (model $B$ ). Regarding model $B$, the uncertainty on the size $L$ of the magnetic halo affects the limits up to one order of magnitude as represented by the red band in Fig 3. For both propagation models, PBHs with masses smaller than $10^{16} \mathrm{~g}$ cannot contribute more than $0.1 \%$ to the DM density of the Galaxy. 

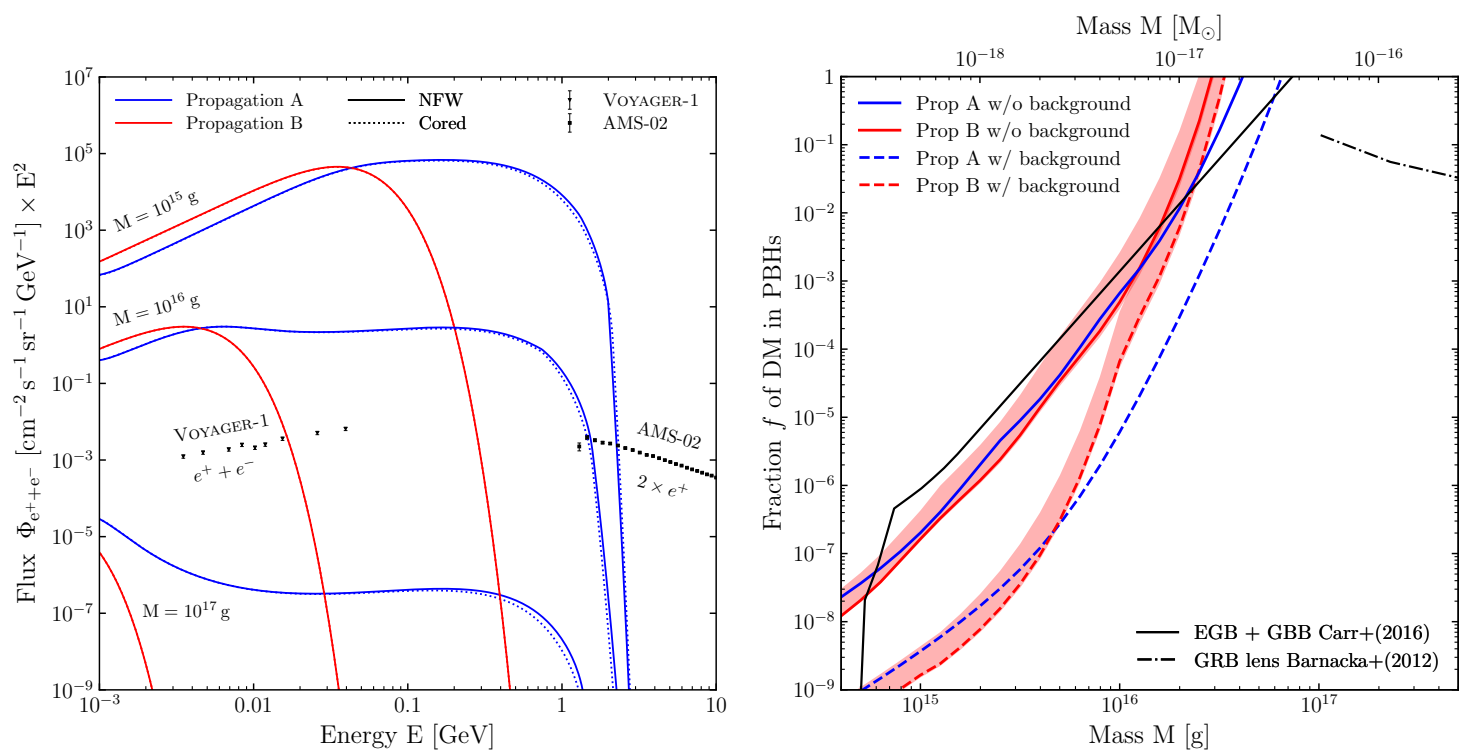

Figure 3: Left: spectra of $e^{ \pm}$from PBH evaporation, after propagation in the Galaxy, for different PBH masses and with the indicated assumptions. The Voyager-1 and AMS-02 data are also reported (the error bars are so small on this scale that they are included in the size of the point). Right: constraints on the fraction $f$ of PBHs to the DM as a function of the PBH mass, as obtained in this work (blue and red lines) and in related studies (black lines) assuming a single mass common to all PBHs.

\section{Conclusion}

We have used the electron and positron data from Voyager-1 to probe locally the DM. We derive limits on the DM particles annihilation cross-section ( $s$-wave and $p$-wave) or decay lifetime. For $s$-wave annihilation, we get limits down to $\langle\sigma v\rangle \sim 10^{-28} \mathrm{~cm}^{3} / \mathrm{s}$ at $10 \mathrm{MeV}$, quite competitive with respect to complementary gamma-ray studies, and less dependent on the halo shape. Limits for $p$-wave annihilation are much more stringent than those derived from complementary astrophysical messengers in the MeV-TeV energy range. We also derive limits on the fraction of DM in the form of PBHs. We found that Voyager-1 is sensitive to a signal from PBHs with $M \lesssim 10^{16} \mathrm{~g}$. We therefore constrained the fraction of PBHs to the DM density to be smaller than $0.1 \%$ for $M \lesssim$ $10^{16} \mathrm{~g}$. We also showed that considering a log-normal mass distribution (as predicted by inflationary models) significantly improves the constraints. Our limits are competitive with those derived from cosmological observations. These limits are robust regarding the DM distribution in the Galaxy and they are not affected by solar activity, precisely because Voyager-1 data have been collected beyond the heliopause. We estimate the propagation uncertainty on our limits to be around one order of magnitude. We emphasize that these new limits are based on local measurements and do not depend on any cosmological parameters. They are therefore fully complementary to other limits derived from cosmological observations.

\section{Acknowledgement}

This proceeding is a summary of several analyses I performed with my collaborators for whom I am very grateful: Marco Cirelli, Thomas Lacroix, Julien Lavalle, Pierre Salati and Martin Stref. 


\section{References}

[1] A. C. Cummings, E. C. Stone, B. C. Heikkila, N. Lal, W. R. Webber, G. Jóhannesson et al., Galactic Cosmic Rays in the Local Interstellar Medium: Voyager 1 Observations and Model Results, Astrophys. J. 831 (2016) 18.

[2] M. Boudaud, J. Lavalle and P. Salati, Novel cosmic-ray electron and positron constraints on MeV dark matter particles, Phys. Rev. Lett. 119 (2017) 021103 [1612 .07698].

[3] M. Boudaud, T. Lacroix, M. Stref and J. Lavalle, Robust cosmic-ray constraints on p-wave annihilating MeV dark matter, Phys. Rev. D99 (2019) 061302 [1810 . 01680 ].

[4] M. Boudaud and M. Cirelli, Voyager $1 e^{ \pm}$Further Constrain Primordial Black Holes as Dark Matter, Phys. Rev. Lett. 122 (2019) 041104 [1807.03075].

[5] J. F. Navarro, C. S. Frenk and S. D. M. White, A universal density profile from hierarchical clustering, The Astrophysical Journal 490 (1997) 493.

[6] P. J. McMillan, The mass distribution and gravitational potential of the Milky Way, MNRAS 465 (2017) 76 [1608.00971].

[7] D. Maurin, F. Donato, R. Taillet and P. Salati, Cosmic rays below $z=30$ in a diffusion model: new constraints on propagation parameters, Astrophys. J. 555 (2001) 585 [0101231].

[8] M. Boudaud, E. F. Bueno, S. Caroff, Y. Genolini, V. Poulin, V. Poireau et al., The pinching method for Galactic cosmic ray positrons: implications in the light of precision measurements, Astron. Astrophys. 605 (2017) A17 [1612.03924].

[9] F. Donato, N. Fornengo, D. Maurin and P. Salati, Antiprotons in cosmic rays from neutralino annihilation, Phys. Rev. D69 (2004) 063501 [0 306207 ].

[10] A. Reinert and M. W. Winkler, A Precision Search for WIMPs with Charged Cosmic Rays, JCAP 1801 (2018) 055 [1712.00002].

[11] T. Lacroix, M. Stref and J. Lavalle, Anatomy of eddington-like inversion methods in the context of dark matter searches, JCAP 09 (2018) 040 [1805.02403].

[12] T. R. Slatyer, Indirect dark matter signatures in the cosmic dark ages. I. Generalizing the bound on $s$ -wave dark matter annihilation from Planck results, Phys. Rev. D 93 (2016) 023527 [1506. 03811 ].

[13] H. Liu, T. R. Slatyer and J. Zavala, Contributions to cosmic reionization from dark matter annihilation and decay, Phys. Rev. D 94 (2016) 063507 [1604.02457].

[14] Y. Akrami, F. Arroja, M. Ashdown, J. Aumont, C. Baccigalupi, M. Ballardini et al., Planck 2018 results. 1. overview and the cosmological legacy of planck, ArXiv e-prints (2018) [1807.06205].

[15] R. Essig, E. Kuflik, S. D. McDermott, T. Volansky and K. M. Zurek, Constraining light dark matter with diffuse X-ray and gamma-ray observations, Journal of High Energy Physics 11 (2013) 193 [1309.4091].

[16] A. Massari, E. Izaguirre, R. Essig, A. Albert, E. Bloom and G. A. Gómez-Vargas, Strong optimized conservative fermi-lat constraints on dark matter models from the inclusive photon spectrum, Phys. Rev. D 91 (2015) 083539 [1503.07169].

[17] Y. Zhao, X.-J. Bi, H.-Y. Jia, P.-F. Yin and F.-R. Zhu, Constraint on the velocity dependent dark matter annihilation cross section from fermi-lat observations of dwarf galaxies, Phys. Rev. D 93 (2016) 083513 [1601.02181]. 
[18] P. J. McMillan, The mass distribution and gravitational potential of the Milky Way, MNRAS 465 (2017) 76 [1608.00971].

[19] B. J. Carr, K. Kohri, Y. Sendouda and J. Yokoyama, New cosmological constraints on primordial black holes, Phys. Rev. D81 (2010) 104019 [0912.5297]. 